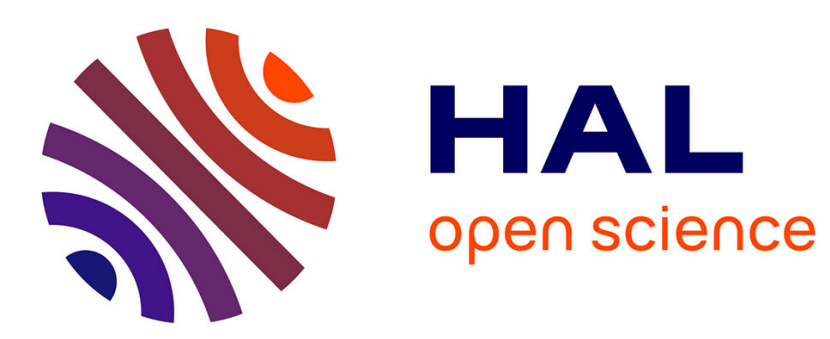

\title{
Observations pédologiques sur le Quaternaire du Lauragais toulousain
}

Jacques Hubschman

\section{To cite this version:}

Jacques Hubschman. Observations pédologiques sur le Quaternaire du Lauragais toulousain. Bulletin de l'Association française pour l'étude du quaternaire, 1972, 9 (1), pp.31-49. 10.3406/quate.1972.1191 . hal-02736314

\section{HAL Id: hal-02736314 \\ https://hal-univ-tlse2.archives-ouvertes.fr/hal-02736314}

Submitted on 2 Jun 2020

HAL is a multi-disciplinary open access archive for the deposit and dissemination of scientific research documents, whether they are published or not. The documents may come from teaching and research institutions in France or abroad, or from public or private research centers.
L'archive ouverte pluridisciplinaire $\mathbf{H A L}$, est destinée au dépôt et à la diffusion de documents scientifiques de niveau recherche, publiés ou non, émanant des établissements d'enseignement et de recherche français ou étrangers, des laboratoires publics ou privés. 


\title{
Observations pédologiques sur le Quaternaire du Lauragais
} toulousain

\author{
Jacques Hubschman
}

\section{Citer ce document / Cite this document :}

Hubschman Jacques. Observations pédologiques sur le Quaternaire du Lauragais toulousain. In: Bulletin de l'Association française pour l'étude du quaternaire, vol. 9, n¹, 1972. pp. 31-49;

doi : https://doi.org/10.3406/quate.1972.1191

https://www.persee.fr/doc/quate_0004-5500_1972_num_9_1_1191

Fichier pdf généré le 19/04/2018 


\begin{abstract}
In the hills of the Lauragais region near Toulouse, we can see high surfaces surrounding slopes covered with colluvium and lœss deposits in the hollows. The existence of a lehm as been noticed and its characters have been analysed. The pedological study of the different morphological units allows us to precise the age the origine of the formations and to draw up a chronological scheme of the quaternary evolution. The following conclusions can be formulated : - The higt surfaces are not the remains of very ancient levels, but they have been recently shapened, probably during the first part of Würm. - The slopes colluvium is younger than the lœss deposit and dating from the end of Würm or beginning of Holocene. It has been built out of the destruction of the high surfaces.
\end{abstract}

\title{
Résumé
}

Les coteaux molassiques du Lauragais toulousain montrent une alternance de replats dominants, de versants tapissés de colluvions et de placages lœssiques. L'existence d'un lehm est signalée et ses caractères sont analyses. L'étude pédologique des diverses unités morphologiques permet de préciser l'âge et l'origine des formations et d'esquisser un schéma chronologique de l'évolution quaternaire. On en tire les principales conclusions suivantes : - Les replats dominants ne sont pas des vestiges de surface ancienne, mais ont été taillés à une époque récente, antérieurement au dépôt du lœss, et datent probablement de la première partie du Würm. - Les colluvions qui drapent les versants, postérieures au dépôt du lœss, datent de l'extrême fin du Würm ou du début de l'Holocène. Elles tirent leur origine de la destruction partielle des replats dominants. 


\title{
OBSERVATIONS PEDOLOGIQUES SUR LE QUATERNAIRE DU LAURAGAIS TOULOUSAIN *
}

\author{
Jacques HUBSCHMAN, \\ Institut de Géographie, \\ Université de Toulouse - Le Mirail.
}

\begin{abstract}
Résumé. - Les coteaux molassiques du Lauragais toulousain montrent une alternance de replats dominants, de versants tapissés de colluvions et de placages loessiques. L'existence d'un lehm est signalée et ses caractères sont analysés. L'étude pédologique des diverses unités morphologiques permet de préciser l'âge et l'origine des formations et d'esquisser un schéma chronologique de l'évolution quaternaire. On en tire les principales conclusions suivantes :

- Les replats dominants ne sont pas des vestiges de surface ancienne, mais ont été taillés à une époque récente, antérieurement au dépôt du loss, et datent probablement de la première partie du Wïrm.

- Les colluvions qui drapent les versants, postérieures au dépôt du loss, datent de l'extrême fin du Würm ou du début de l'Holocène. Elles tirent leur origine de la destruction partielle des replats dominants.
\end{abstract}

Summary. - In the hills of the Lauragais region near Toulouse, we can see high surfaces surrounding slopes covered with colluvium and loess deposits in the hollows. The existence of a lehm as been noticed and its characters have been analysed. The pedological study of the different morphological units allows us to precise the age the origine of the formations and to draw up a chronological scheme of the quaternary evolution. The following conclusions can be formulated:

- The higt surfaces are not the remains of very ancient levels, but they have been recently shapened, probably during the first part of Würm.

- The slopes colluvium is younger than the loss deposit and dating from the end of Wiirm or beginning of Holocene. It has been built out of the destruction of the high surfaces.

Deux ensembles majeurs s'opposent en Aquitaine du sud-est (fig. 1):

- d'une part, les couloirs alluviaux de la Garonne et de l'Ariège : vastes horizons plats des terrasses, dominés par les hauts niveaux des cailloutis supérieurs; - d'autre part, les côteaux de Terrefort du Lauragais, du Savès et de l'Ariégeois, sculptés dans la molasse oligocène et miocène, entaillés de petites vallées généralement dissymétriques.

Dans les grandes vallées, terrasses, cailloutis et limons constituent d'évidence un matériel de choix pour l'étude du Quaternaire. En revanche, les interfluves molassiques, intensément disséqués par un dense chevelu hydrographique et différenciés de ce fait en une foule de paysages élémentaires, représentent un domaine apparemment moins favorisé à cet égard.

Le modelé du Terrefort révèle néanmoins, d'une façon très générale, les traces de plusieurs épisodes anciens: niveaux d'aplanissement, dépôts éoliens. colluvions de versant, ont été décrits dans la plupart des travaux antérieurs

* Manuscrit déposé le 15 novembre 1971. 


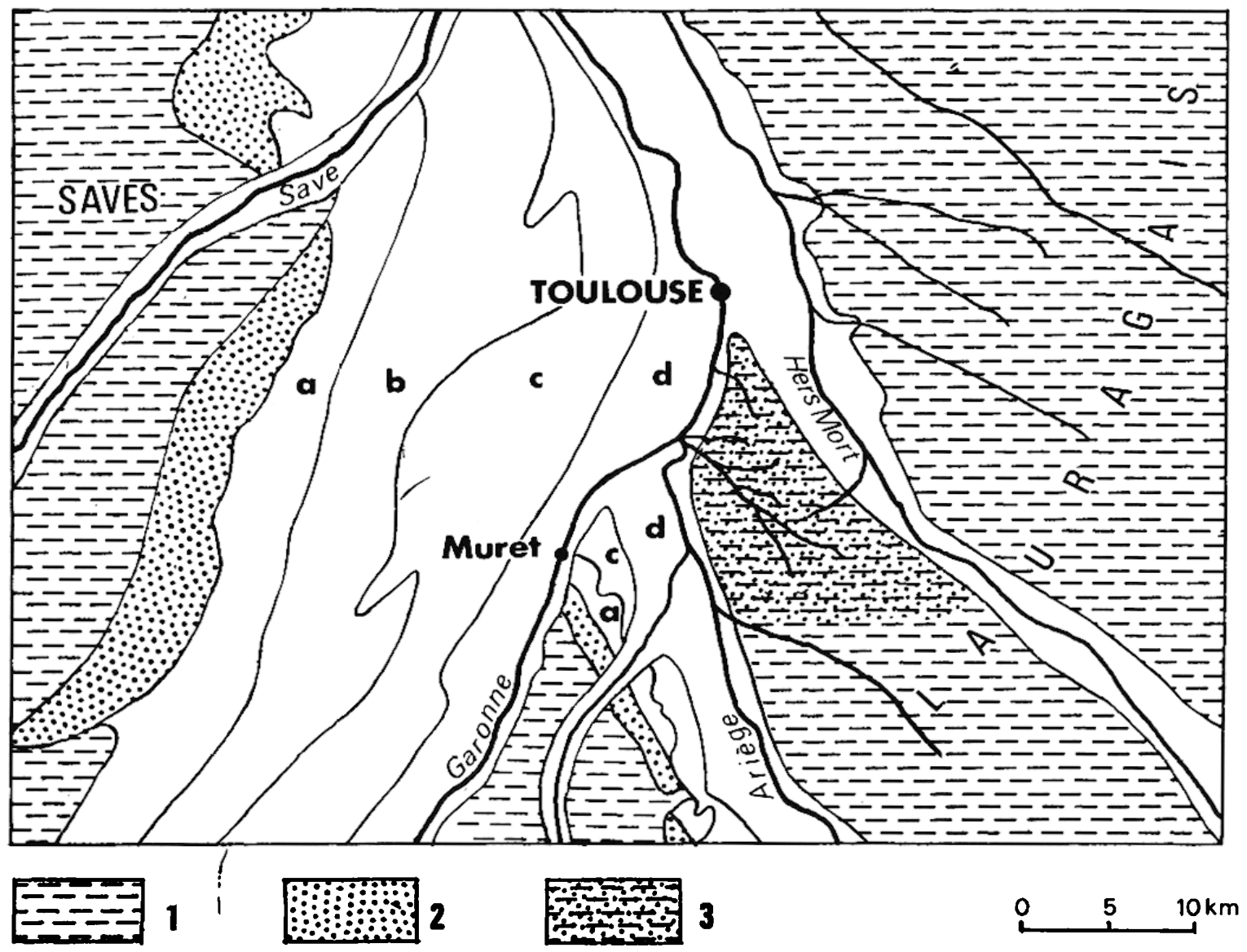

Fic. 1. - Pays de Terrefort et plaines alluviales dans la région toulousaine.

1. Terrefort molassique. - 2. Hauts niveaux des cailloutis garonnais et ariégeois. - 3. Secteur étudié.

a. Haute terrasse. - b. Terrasse moyenne. - c. Basse terrasse. - d. Basse plaine.

(G. Astre, 1953a; A. Cavaille 1958; G. Astre, 1959; H. Enjalbert, 1960; A. Cavaille, 1969). Certains des principaux problèmes liés à cette morphologie n'ont toutefois pas toujours été pris en considération, ni interprétés de façon identique : âge et état de conservation des hautes surfaces, âge et origine des dépôts, chronologie des événements. Nous avons cherché à les aborder sur de nouvelles bases, en regroupant les principaux éléments d'une étude pédologique conduite dans un secteur particulier du Terrefort. Nous nous proposons ici l'examen de quelques profils représentatifs des grandes unités morphologiques. Nous en tirerons argument pour préciser les rapports entre les formes, les dépôts et leur évolution pédologique. On tentera par la suite d'établir une chronologie sommaire des événements survenus au Quaternaire récent.

\section{I. - APERÇU General}

Le secteur étudié s'étend au sud-est de Toulouse, entre la zone de confluence de l'Ariège-Garonne et la vallée de l'Hers mort (fig. 1). Ce choix est motivé par l'absence presque totale de replats structuraux donnés par les bancs calcaires 


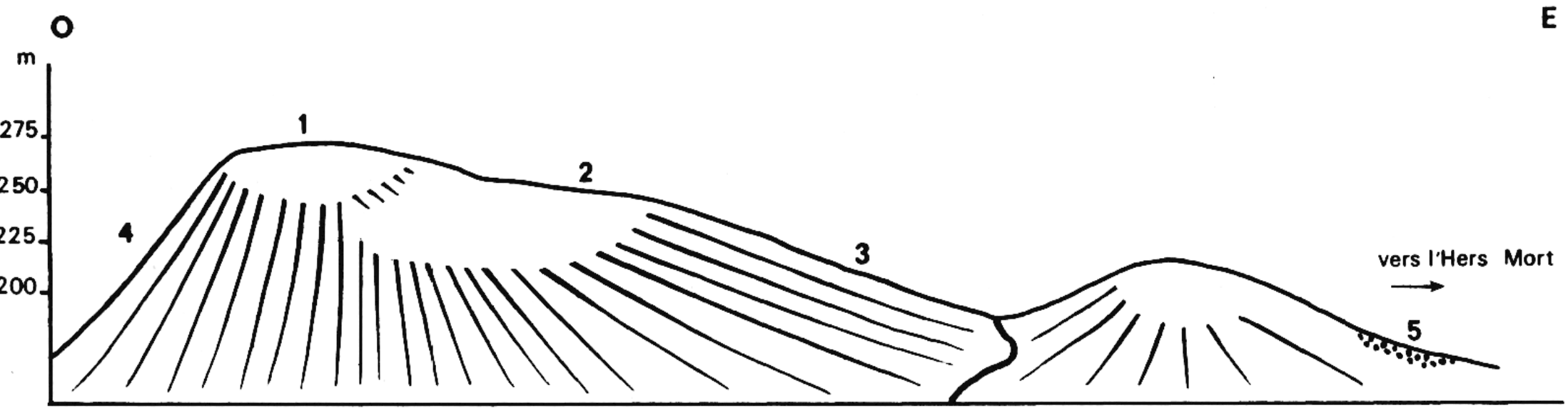

FIG. 2. - Disposition morphologique en Lauragais toulousain. 1. Replat superieur. - 2. Replat inférieur. -3 . Versant long. -4 . Versant 
qui arment parfois la molasse, surtout dans le Terrefort ariégeois, mais aussi au nord-est de Toulouse et dans la Savès.

La morphologie de cette région s'ordonne autour de trois caractères essentiels (fig. 2):

- Les éléments de plateau, où l'on observe couramment deux générations de formes :

- des replats supérieurs, représentés par d'étroits lambeaux (les "serres ») disséqués et allongés dans une direction généralement sud-est-nord-ouest, culminant vers $265-280 \mathrm{~m}$. Les surfaces sommitales sont loin d'être rigoureusement planes; leur allure est plutôt celle de * dos de baleine " très surbaissés souvent inclinés vers le nord-ouest.

- Des replats inférieurs, inscrits sous la surface précédente à environ $240-260 \mathrm{~m}$, plus nettement inclinés vers l'aval que les replats culminants. Leurs limites sont cependant beaucoup plus floues, car bien souvent elles se raccordent très progressivement aux niveaux supérieurs et, vers le bas, aux versants longs à pente modérée. Aussi les côtes d'altitude semblent-elles encore plus variables que celles des surfaces culminantes.

- Entaillant les niveaux précédents, les vallons fréquemment dissymétriques des petits ruisseaux montrent des versants longs à pente modérée, généralement exposés vers l'est. Dans la plupart des cas, ces versants longs sont tapissés de colluvions qui s'épaississent au pied des pentes, remblayant les talwegs et masquant les basses terrasses des rivières secondaires (Hers Mort). Ces dépôts ne s'observent pas sur les versants courts à pente raide, taillés dans la molasse.

- Des placages de loess plus ou moins puissants jalonnent les bas versants molassiques dominant la vallée de l'Hers Mort. Localement, ce loess colmate certains fonds de vallons à l'intérieur même du Terrefort: il est alors fossilisé par des matériaux colluvionnés.

Au tro:s ensembles morphologiques sommairement décrits ci-dessus, répondent les trois catégories de sols auxquels nous limiterons notre étude : sols des plateaux, sols des versants longs, sol sur loess. Un sort particulier sera réservé à ce dernier: c'est en effet, à notre connaissance, l'unique exemple d'un lehm sensu stricto dans la région toulousaine, développé de surcroît sur un matériau relativement bien daté. Nous l'examinerons en premier lieu.

\section{II. - LE LEHM DE TOULOUSE-RANGUEIL}

Le loess se présente en placages discontinus généralement épais ( 3 à $8 \mathrm{~m}$ ), de couleur jaunâtre, à structure massive très homogène, se débitant par grands pans verticaux. La distribution des sables est typique d'un matériel éolien ( $80 \%$ des particules comprises entre $0,050 \mathrm{~mm}$ et $0,125 \mathrm{~mm})^{1}$. Il est riche en calcaire (10 à $25 \%)$ et les carbonates y revêtent plusieurs aspects : formes diffuses (calcaire fin et très fin) ou nettement individualisées ( ( points blancs ", pseudomycélium, taches et fissures à calcaire pulvérulent, vermicules et tubulures, éléments de racines pétrifiées). Par place, on observe des concrétions dures, nodules arrondis ou poupées d'aspect tourmenté, souvent creux et à cristallisat:on rayonnante. L'ensemble des coupes ne montre qu'une seule génération de dépôts éoliens: ceux-ci ont pu être datés par leur faune molacologique au Würm supérieur (A. CAvaIlle, 1969).

1. Avec prédominance de sables fins. 
Dans les travaux antérieurs (E. Chaput, 1928; G. Astre, 1956; A. Cavaille, 1969), aucune mention n'est faite de sols développés sur le loess. Celui-ci, il est vrai, est fréquemment plaqué contre des versants déjà raides, situation défavorable à la pédogénèse et fort propice à l'érosion. Aussi bien le loess qui tapisse certains fonds de vallons ne présente-t-il jamais la moindre altération superficielle: les colluvions des pieds de versants recouvrent toujours un matériel d'aspect très frais. C'est dire que, dans ces conditions, seul un site particulièrement favorable autorisait le développement et la conservation d'un sol établi sur le loess: pente modéré, ruissellement d'amont déficient ou dévié vers un proche talweg... Nous avons pu en retrouver un exemple à proximité de Toulouse (face au complexe scientifique de Rangueil), où une carrière nous a livré le profil d'un épais sol sur loess. Dans une note récente (J. HubschmaN, 1971), nous avons décrit les conditions de gisement, l'allure générale ainsi que les caractères essentiels de ce lehm, dont l'étude sédimentologique révèle la parfaite filiation avec le loess. Nous en donnons ci-dessous une description sommaire.
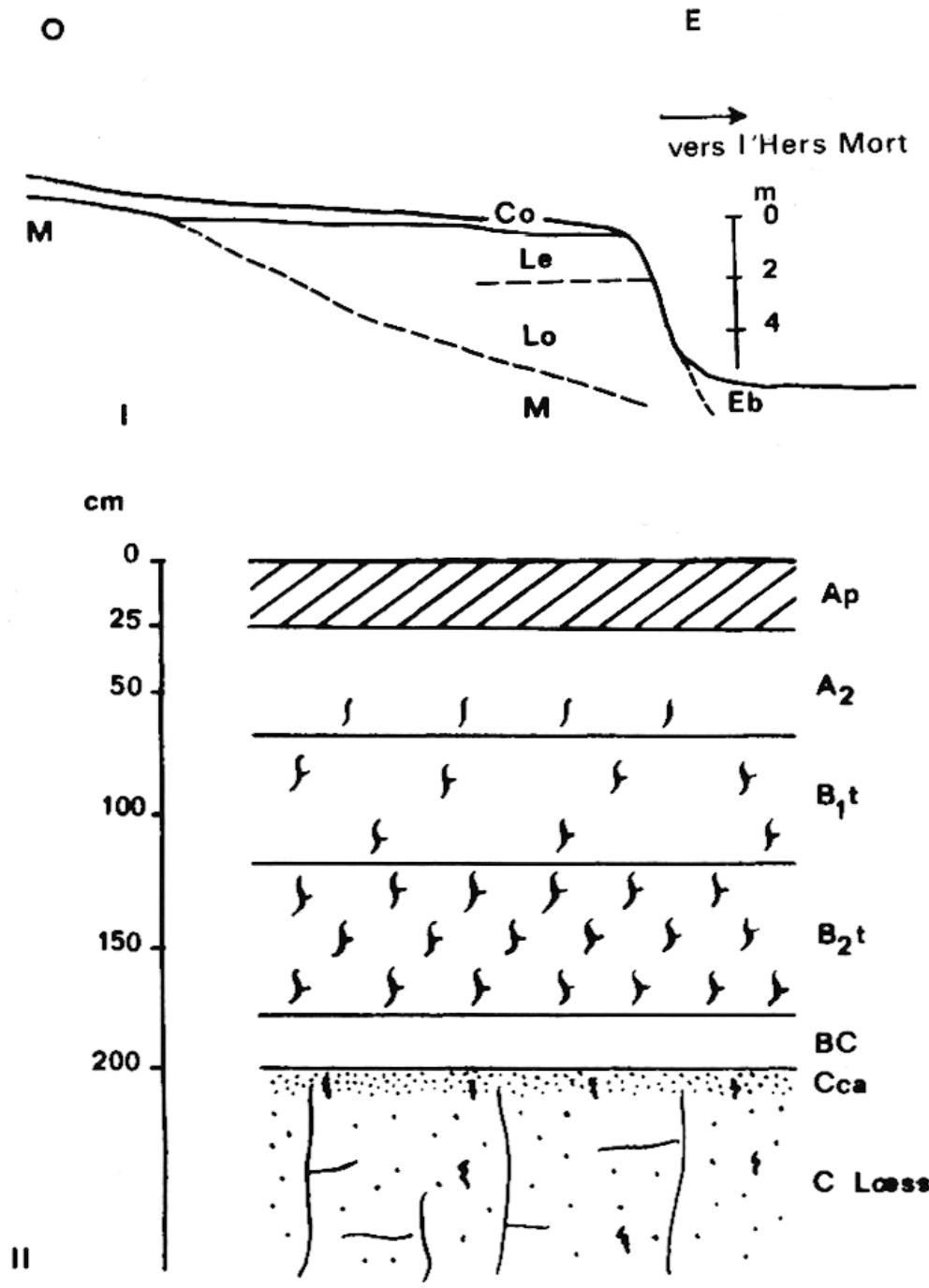

Fig. 3. - Le lehm de Toulouse-Rangueil.

I. Site. M. Molasse. - Co. Colluvium et remaniement récent. - Le. Lehm. Lo. Loess. - Eb. Eboulis.

II. Profil du lehm (description dans le texte). 
Profil pédologtoue du lehm (fig. 3-11)

Situation (fig. $3-1)$ : pente faible (7-8 \%) vers la vallée de l'Hers-Mort. Vieille prairie abandonnée (Graminées).

$0 \cdot 25$

Ap- $\mathbf{A}_{1}$

$\mathrm{cm}$ : brun sombre, plus clair en profondeur, nombreuses racines

$25-70$

$A_{2}$ puis

$A_{2}-B, t$

$70-120$

$\mathbf{B}_{1} \mathbf{t}$

$120-170$

$\mathbf{B}_{\mathbf{2}} \mathrm{t}$ fines, structure grenue, non calcaire, limites assez nettes avec $A_{2}$;

$\mathrm{cm}$ : brun clair $10 \mathrm{YR} 6 / 3$, homogène, structure légèrement massive à tendance polyédrique plus nette avec la profondeur, non calcaire, limites très progressives avec $\mathrm{B}_{2} \mathrm{t}$;

$\mathrm{cm}$ : brun 10 YR $6 / 3$ à 10 YR $5 / 4$, structure polyédrique nette, quelques revêtements argileux plus foncés à la surface des agrégats, non calcaire, limites très progressives avec $B_{2} t$;

$170-200$

B C

$\mathrm{cm}$ : brun 10 YR $5 / 4$ avec d'étroites zones verticales à 7 YR 5/4, correspondant aux revêtements argileux assez abondants, non calcaires, limites progressives avec $\mathrm{BC}$;

$200-220$

Cca

$\mathrm{cm}$ : brun-jaune clair 2,5 YR $6 / 4$ à 10 YR $7 / 6$, structure massive très homogène, non calcaire, limites assez nettes avec $\mathrm{C}$ ca;

Plus de $220 \mathrm{~cm}$ : formation loessique typique, calcaire, se débitant en gros blocs C réguliers, pseudomycéliums et taches quelques poupées et concrétions calcaires, visible jusqu’à $5 \mathrm{~m}$ environ.

\section{TABLEAU 1}

\section{Données analytiques (Lehm) *}

\begin{tabular}{|c|c|c|c|c|c|c|c|c|c|c|}
\hline \multirow{2}{*}{$\begin{array}{l}\text { Profondeur } \\
\text { (cm) }\end{array}$} & \multicolumn{5}{|c|}{ Granulométrie (z) } & \multicolumn{2}{|c|}{ Calcairc (z) } & \multicolumn{2}{|c|}{$\mathrm{pH}$} & \multirow{2}{*}{$c(x)$} \\
\hline & SG & SF & LG & LF & $\mathbf{A}$ & $T$ & $\mathbf{a}$ & eau & $\mathrm{RCl}$ & \\
\hline $30-40$ & 1,7 & 48,0 & 18,5 & 10,7 & 19,7 & 0,7 & 0 & 8,3 & 7,2 & 0,39 \\
\hline $70-80$ & 1,7 & 47,7 & 17,0 & 9,9 & 21,9 & 0,2 & 0 & 8,0 & 7,0 & 0,39 \\
\hline $140-150$ & 1,8 & 48,9 & 13,8 & 9,5 & 25,2 & 0,2 & 0 & 7,9 & 6,9 & 0,34 \\
\hline $180-200$ & 1,9 & 52,2 & 13,3 & 9,3 & 22,7 & 0,2 & 0 & 8,2 & 7,0 & 0,18 \\
\hline $200-220$ & & & & & & 19,9 & 3,4 & 8,7 & 7,6 & 0,18 \\
\hline $250-260$ & 3,8 & 61,6 & 14,0 & 7,8 & 12,4 & 12,7 & 2,0 & 8,7 & 7,7 & 0,19 \\
\hline
\end{tabular}




\begin{tabular}{|c|c|c|c|c|c|c|c|c|c|}
\hline \multirow{2}{*}{$\begin{array}{l}\text { Profondeur } \\
(\mathrm{cm})\end{array}$} & \multicolumn{4}{|c|}{ Bases échang. m. e. \% } & \multirow{2}{*}{$\begin{array}{c}\mathrm{S} \\
\mathrm{m} \cdot \mathrm{e} \cdot \mathrm{z}\end{array}$} & \multirow{2}{*}{$\begin{array}{c}\mathrm{T} \\
\mathrm{m} \cdot \mathrm{e} . \%\end{array}$} & \multirow{2}{*}{$\frac{S}{T} \not$} & \multirow{2}{*}{$\underset{\pi}{\mathrm{FeT}}$} & \multirow{2}{*}{$\begin{array}{c}\text { FeL } \\
\pi\end{array}$} \\
\hline & $\mathrm{Ca}$ & $\mathrm{K}$ & $\mathrm{Mg}$ & $\mathrm{Na}$ & & & & & \\
\hline $30-40$ & 19,3 & 0,13 & 0,60 & 0,07 & & 9,9 & 100 & 1,19 & 0,56 \\
\hline $70-80$ & & & & & & & & & \\
\hline $140-150$ & 16,8 & 0,21 & 0,40 & 0,07 & & 13,5 & 100 & 1,54 & 0,76 \\
\hline $180-200$ & 14,3 & 0,19 & 0,35 & 0,07 & & 12,1 & 100 & 1,47 & 0,78 \\
\hline $200-220$ & & & & & & & & & \\
\hline $250-260$ & 38,0 & 0,09 & 0,35 & 0,07 & & 6,7 & 100 & 0,88 & 0,32 \\
\hline
\end{tabular}

\begin{tabular}{|c|c|c|c|c|c|}
\hline Profondeur & $30-40$ & $140-150$ & $180-200$ & $200-220$ & $250-260$ \\
\hline Illite & 30 & 30 & 25 & 25 & 20 \\
\hline Montmorillonite & 10 & 30 & 40 & 40 & 60 \\
\hline Kaolinite & 20 & 10 & 10 & 15 & 15 \\
\hline Chlorite & & tr. (?) & tr. (?) & 5 & 5 \\
\hline Interstratifiés & 30 & 30 & 25 & 15 & \\
\hline $\begin{array}{l}\text { Interstratifies } \\
(14 \mathrm{C}-14 \mathrm{M})\end{array}$ & $10(?)$ & & & & \\
\hline
\end{tabular}

* S.G. : sables grossiers de $0,200 \mathrm{~mm}$ à $2 \mathrm{~mm}$; S.F. : sables fins de $0,050 \mathrm{~mm}$ à $0,200 \mathrm{~mm}$; L.G. : limons grossiers de $0,020 \mathrm{~mm}$ à $0,050 \mathrm{~mm}$; L.F. : limons fins de $0,002 \mathrm{~mm}$ à $0,020 \mathrm{~mm}$; A : argile inférieure à $0,002 \mathrm{~mm}$. Calcaire $T$ : total ; a : fin (actif).

C : carbone organique.

$\mathrm{S}$ : somme des bases échangeables en milliéquivalents pour $100 \mathrm{~g}$ (m.e. \%).

T : capacité totale d'échange des bases en m.e. \%.

S/T : taux de saturation du complexe absorbant.

Fe T : fer total (acide chlorhydrique concentré, technique O.R.S.T.O.M.).

Fe L : fer libre (méthode Deb modifiée O.R.S.T.O.M.)

Minéraux argileux : diffraction des rayons $X$, analyse thermique differentielle; estimation des proportions pour un stock total de $\mathbf{1 0 0}$. 


\section{Commentaires :}

- l'épaisseur du lehm atteint près de $200 \mathrm{~cm}, 175 \mathrm{~cm}$ si l'on fait abstraction de l'horizon Ap qui provient en partie d'un remaniement récent lié essentiellement à la mise en culture. On observe au contact du loess un horizon d'accumulation de calcaire fin.

- Le lehm correspond à un sol brun lessivé. Le lessivage demeure toutefois modéré : les indices de distribution d'argile et de fer libre ne dépassent pas 1,3. Les enrobements argileux sont cependant très nets $B_{1} t$ et $B_{2} t$.

- Le milieu est basique, le complexe absorbant totalement saturé (surtout en calcium) et le sol contient encore des traces de calcaire : on sait maintenant (P. Duchaufour, 1968) que le lessivage peut se manifester dans de telles conditions (sol brun calcique lessivé).

- Le stock de minéraux argileux du loess est riche en montmorillonite; illite et kaolinite sont subordonnées, la chlorite est fort peu abondante. Ce mélange mime assez fidèlement la composition de divers échantillons de molasse que nous avons prélevés sur les hauteurs environnantes du Terrefort (en moyenne : montmorillonite 50 , illite 30 , kaolinite 20 , traces de chlorite ${ }^{1}$ ). C'est la preuve que le loess tire son origine de la molasse et qu'il n'est sans doute pas venu de très loin. L'évolution des argiles est conforme au schéma classique évoqué par T. CAMrez (1962) pour des sols encore jeunes et peu acides. La montmorillonite diminue en surface au bénéfice des interstratifiés qui apparaissent tôt dans le profil, et de l'illite. La kaolinite semble assez variable, la chlorite peu abondante disparaît rapidement.

Développé à partir d'un matériel bien daté, le lehm témoigne d'un degré d'altération spécifique. Compte tenu de la diversité des sites et des roches-mères, l'évolution du loess pourra être confrontée à celle d'autres formations du Terrefort et, en particulier, à la pédogenèse des sols de plateau.

\section{III. - Les elements de PLATEAuX et les SOlS}

L'existence des aplanissements supérieurs, qui rythment la topographie du Terrefort, a été diversement interprétée suivant les auteurs. Certains en ont fait l'équivalent de la surface néogène définie dans le Terrefort tarnais (G. ASTRE, 1953b ; H. EnJalbert, 1960). Pour R. Brunet (1965) ces aplanissements pourraient être contemporains des glacis du Pliocène et du Quaternaire ancien auxquels H. ENJalbert, assimile les hauts niveaux garonnais et ariégeois. A. Cavaillé (1969), se fondant sur la présence de certains affleurements de quartz rougi sur les replats supérieurs y voit les traces d'un aplanissement pliocène. Ces niveaux sont donc généralement considérés comme des témoins de surfaces très anciennes que l'érosion quaternaire a réduit en lambeaux. La différence d'altitude que l'on observe actuellement entre les hauts replats du Lauragais $(275 \mathrm{~m}$ en moyenne dans le secteur étudié) et les cailloutis anciens garonnais et ariégeois $(310 \mathrm{~m}$ à l'ouest de Toulouse et au sud de Muret) a pu être attribuée à un affaissement par flexures et gauchissements récents du Lauragais (H. ENJALBERT 1960). L'étude pédologique des sols de plateau nous conduit à une toute autre interprétation.

Avant d'aborder l'étude des sols de plateau et afin de ne laisser place à aucun malentendu, il convient de spécifier le champ d'application et l'échelle

\footnotetext{
1. Les fortes concentrations de montmorillonite dans la molasse corroborent l'existence de très fréquents paléosols tertiaires intercalés dans les formations oligocènes et miocènes. que nous avons pu observer en compagnie de F. CROUzEt. Ces paléosols tertaires feront l'objet d'une note ultérieure.
} 
de nos observations. Dans le Lauragais, en effet, volumes en relief des coteaux molassiques et volumes en creux des versants et vallons, constituent les grands traits du modelé : il est clair que, pour l'essentiel, cette disposition représente un héritage du Quaternaire ancien ou, tout au moins, d'une période préglaciaire (au sens * pyrénéen " de ce terme). Il reste cependant que l'érosion qui s'est exercée au cours des phases ultérieures a très sensiblement modifié le modelé ancien. C'est précisément à des phénomènes de cette échelle que s'applique l'étude des sols de plateau: elle permet à la fois d'apprécier l'ampleur du remaniement et de dater le dernier façonnement effectif des surfaces sommitales.

\section{LES SOLS (fig. 4-1 et II)}

Les " formations résiduelles des plateaux ", connues parfois sous le nom de " boulbènes de plateau n, tapissent régulièrement les replats du Terrefort. Nivcaux supérieurs et niveaux inférieurs montrent des sols dont les caractères morphologiques et analytiques sont identiques. On notera toutefois que la partie amont des replats inférieurs, lorsque ceux-ci se trouvent effectivement dominés par un replat supérieur, porte bien souvent un dépôt colluvionné qui s'apparente à ceux qui tapissent les versants longs. Ce dépot recouvre alors soit la molasse, soit un mince éluvium de celle-ci.

D'une épaisseur moyenne de $1 \mathrm{~m}$ à $1,50 \mathrm{~m}$, les sols de plateau reposent sur la molasse stampienne dont ils dérivent directement. C'est une roche-mère très hétérogène, dont l'aspect et la composition varient largement d'un point à l'autre : aussi les sols reflètent-ils très fidèlement ces différences de faciès. On peut. à cet égard, distinguer les deux principaux types de molasse :

- un faciès argilo-calcaire, à bariolage orange et gris-bleu souvent disposé en strates sub-horizontales. Le calcaire s'individualise fréquemment soit sous la

a

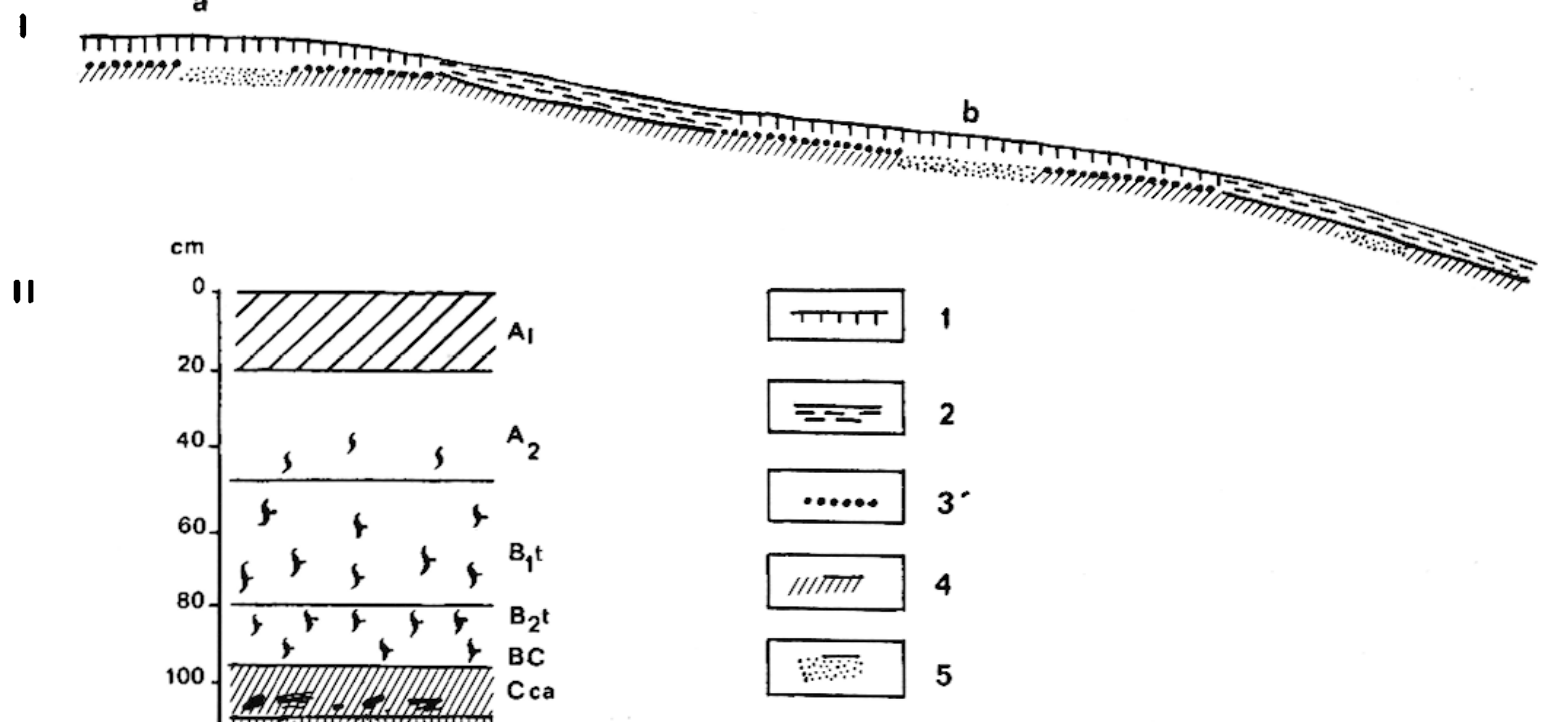

Fic. 4. - Les sols de plateau.

-1 . Sols de plateaux. -2 . Sols bruns calcaires et bruns calciques sur colluvions récentes. - 3. Horizon d'accumulation calcaire. - 4. Faciès argilo-calcaire de la molasse. - 5. Faciès sableux non calcaire de la molasse.

II. Profil d'un sol de plateau sur molasse argilo-calcaire (sol brun calcique à profil textural différencié, description dans le texte). 
forme de minces lits pulvérulents également sub-horizontaux (ceci, uniquement à la partie supérieure de la molasse, semble-t-il), soit en éléments faiblement consolidés (" têtes de chats", de G. ASTRE). Les sols développés sur un tel matériau présentent régulièrement au contact de la molasse un horizon d'accumulation calcaire clairement apparent dans les talus de chemins creux;

- un faciès à tendance sableuse, non calcaire, riche en taches rouille et ocre. Bien que trés répandue, cette variété de molasse couvre des surfaces moins étendues que la précédente.

Un profil-type de chaque catégorie sera présenté ci-dessous.

Profil 1. - Sol de plateau sur molasse argilo-sableuse (fig. 4 II)

Situation: replat supérieur, altitude $272 \mathrm{~m}$, pente 1 à $3 \%$ vers le Sud-Ouest. Vigne abandonnée.

Précédent cultural : Vigne. Pas d'amendement calcaire connu.

$0-20 \mathrm{~cm}$ : brun $10 \mathrm{YR} 5 / 4$, racines, structure à tendance massive, grenue Ap sur les premiers $\mathrm{cm}$, plus grossière et polyédrique en profondeur, non calcaire, forte activité biologique (pores, déjections), limites progressives avec $A_{2}$;

20-50 cm: brun $10 \mathrm{YR} 4 / 4$, structure massive devenant polyédrique vers $A_{2} \quad$ vers le bas, fentes verticales et horizontales, non calcaire, limites progressives avec $B_{1}$ t;

$50-80 \mathrm{~cm}$ : brun rougeâtre 10 YR $4 / 4$ à 7,5 YR 4/4, plus argileux, structure B.t polyédrique à nette tendance prismatique, nombreux revêtements argileux, non calcaire, limites progressives avec $\mathbf{B}_{2} t$;

80-95 cm: ibidem $B_{1} t$ mais apparition progressive du faciès molassique $\mathrm{B}_{2} \mathrm{t}$ et $\mathrm{BC}$ a zones claires $10 \mathrm{YR} 8 / 1$, structure moins régulière, présence de calcaire diffus en profondeur, limites nettes avec Cca;

95-110 cm: horizon d'accumulation calcaire, couleur crême générale due Cca au carbonate diffus, quelques concrétions dures (diamètre: 1 à $3 \mathrm{~cm}$ ) et poupées creuses, quelques éléments de croûte légèrement feuilletée, disloqués et pourris, passage progressif à la molasse sous-jacente;

Plus de $110 \mathrm{~cm}$ : molasse stampienne à stratifications grises et orangées, à minces lits horizontaux de calcaire pulvérulent (observation jusqu’à $180 \mathrm{~cm}$ ).

\section{Profil 2. - Sol de plateau gur molasse sableuse}

Situation : replat inférieur, altitude $249 \mathrm{~m}$, pente $4 \%$ vers le Nord-Ouest. Jachère sur blé. Précédents culturaux: vigne puis céréales. Pas d'amendement calcaire connu.

$0.20 \mathrm{~cm}$ : brun clair à beige, texture sableuse, racines, structure grenue à nuciforme, nombreuses muscovites visibles, non calcaire, limites progressives avec $\mathbf{A}_{2}$;

20-45 cm: brun-jaune clair, texture sableuse, muscovites nombreuses, $A_{2} \quad$ structure massive, horizon compact, non calcaire, limites progressives avec B,t; 
45-80 cm: brun-jaune plus foncé, texture sablo-argileuse, nombreuses B,t muscovites, structure massive à tendance polyédrique, revêtements argileux nets, horizon compact, quelques petits points de couleur rouille à la base, non calcaire, limites progressives avec $B_{2} t$;

80-105 cm: identique à $B_{1} t$ mais le faciès de la molasse apparaît de plus $B_{2}$ et $\mathrm{BC}$ en plus nettement avec la profondeur: taches rouille et zones plus claires, structure plus massive que $B_{1} t$, limites très progressives avec la molasse;

Plus de $105 \mathrm{~cm}$ : molasse sableuse riche en muscovites, taches ocre-rouille et C plages grisées, structure massive, compact, non calcaire (observation jusqu'à $180 \mathrm{~cm}$ ).

TABLEAU ॥

Données analytiques sommaires (profils 1 et 2)

\begin{tabular}{|c|c|c|c|c|c|c|c|c|c|c|}
\hline \multirow{2}{*}{$\begin{array}{l}\text { Profondeur } \\
\text { (cm) }\end{array}$} & \multicolumn{5}{|c|}{ Granulomêtrie (z) } & \multicolumn{2}{|c|}{ Calcair $\quad(\%)$} & \multicolumn{2}{|c|}{$\mathrm{pH}$} & \multirow{2}{*}{ c $(z)$} \\
\hline & SG & SF & LG & LF & A & $\mathbf{T}$ & $\mathbf{a}$ & eau & $\mathrm{xC1}$ & \\
\hline Profil 1 & & & & & & & & & & \\
\hline $10-20$ & 20900 & 22,4 & 14,0 & 16,8 & 23,3 & 2,7 & 0 & 8,3 & 7,2 & 0,45 \\
\hline $35-45$ & 14,0 & 19,7 & 15,5 & 19,6 & 29,3 & 1,0 & 0 & 8,4 & 7,1 & 0,54 \\
\hline $70-80$ & 7,8 & 13,6 & 12,7 & 23,0 & 41,4 & 0,8 & 0 & 8,3 & 7,0 & 0,39 \\
\hline $100-110$ & 5,6 & 23,8 & 17,8 & 28,7 & 23,6 & 16,9 & 4,6 & 8,4 & 7,2 & 0,24 \\
\hline $140-150$ & & & & & 22,2 & 12,1 & 2,9 & & & \\
\hline Profil 2 & & & & & & & & & & \\
\hline $0-20$ & & & & & 14,1 & 0 & 0 & & & \\
\hline $20-45$ & 27,7 & 29,2 & 15,7 & 12.2 & 14,4 & 0 & 0 & 6,8 & 5,5 & 0,36 \\
\hline $60-80$ & 25,3 & 23,8 & 10,7 & 13,9 & 25,7 & 0 & 0 & 6,7 & 5,8 & 0,32 \\
\hline $85-95$ & 42,7 & 18,1 & 5,9 & 11,9 & 21,0 & 0 & 0 & 6,9 & 5,6 & 0,21 \\
\hline $140-150$ & 42,7 & 21,7 & 5,4 & 12,1 & 17,1 & 0 & 0 & 7,0 & 5,6 & 0,15 \\
\hline
\end{tabular}




\begin{tabular}{|c|c|c|c|c|c|c|c|c|c|}
\hline \multirow{2}{*}{$\begin{array}{l}\text { Profondeur } \\
\text { (cm) }\end{array}$} & \multicolumn{4}{|c|}{ Bases Echang. m. e. $z$} & \multirow{2}{*}{$\begin{array}{c}\text { s } \\
\text { m.e.z }\end{array}$} & \multirow{2}{*}{$\begin{array}{c}\mathrm{T} \\
\mathrm{m} \cdot \mathrm{e} \cdot \mathrm{z}\end{array}$} & \multirow{2}{*}{$\frac{5}{T} x$} & \multirow{2}{*}{$\underset{7}{\mathrm{PeT}}$} & \multirow{2}{*}{ Fel } \\
\hline & $\mathrm{Ca}$ & $\mathbf{x}$ & $\mathrm{Mg}$ & $\mathrm{Na}$ & & & & & \\
\hline \multicolumn{10}{|l|}{ Profil 1} \\
\hline $10-20$ & 35,4 & 0,21 & 1,10 & 0,07 & & 14,5 & 100 & 2,24 & 1,52 \\
\hline $35-45$ & 26,4 & 0,21 & 1,30 & 0,07 & & 15,4 & 100 & 2,36 & 1,45 \\
\hline $70-80$ & 31,1 & 0,32 & 1,35 & 0,13 & & 17,7 & 100 & 3,18 & 1,96 \\
\hline $100-110$ & 42,9 & 0,28 & 1,20 & 0,13 & & 19,9 & 100 & 2,93 & 1,87 \\
\hline \multicolumn{10}{|l|}{$140-150$} \\
\hline \multicolumn{10}{|l|}{ Profi1 2} \\
\hline $20-45$ & 6,30 & 0,15 & 0,05 & 0,07 & 6,57 & 7,8 & 84 & 1,02 & 0,67 \\
\hline $60-80$ & 10,4 & 0,17 & 1,55 & 0,10 & 12,22 & 12,6 & 97 & 1,60 & 0,79 \\
\hline $85-95$ & 13,2 & 0,19 & 2,40 & 0,16 & 15,95 & 16,3 & 98 & 1,62 & 1,02 \\
\hline $140-150$ & 13,9 & 0,21 & 2,35 & 0,16 & 16,60 & 17,1 & 97 & 1,48 & 0,76 \\
\hline
\end{tabular}

TABLEAU II (suite)

Minéraux argileux (estimation)

\begin{tabular}{|c|c|c|c|c|c|c|c|c|c|c|}
\hline \multirow{2}{*}{$\begin{array}{l}\text { Profondeur } \\
\text { (cm) }\end{array}$} & \multicolumn{5}{|c|}{ Profil 1} & \multicolumn{5}{|c|}{ Profil 2} \\
\hline & $\begin{array}{l}10- \\
20\end{array}$ & $\begin{array}{l}35- \\
45\end{array}$ & $\begin{array}{l}70- \\
80\end{array}$ & $\begin{array}{l}100- \\
110\end{array}$ & $\begin{array}{l}140- \\
150\end{array}$ & $\begin{array}{l}0- \\
20\end{array}$ & $\begin{array}{l}20- \\
45\end{array}$ & $\begin{array}{l}60- \\
80\end{array}$ & $\begin{array}{l}85- \\
95\end{array}$ & $\begin{array}{l}140 \\
150\end{array}$ \\
\hline Illite & 35 & 20 & 15 & 15 & 20 & 40 & 30 & 30 & 35 & 35 \\
\hline Montmorillonite & 25 & 30 & 35 & 40 & 50 & 30 & 30 & 40 & 55 & 55 \\
\hline Reolinite & 10 & 25 & 30 & 25 & 20 & 10 & 20 & 30 & 10 & 10 \\
\hline Chlorite & & tr. & 5 & 5 & 10 & & tr. & tr. & tr. & tr. \\
\hline $\begin{array}{l}\text { Interstratifiēs } \\
(14 \mathrm{c}-14 \mathrm{v})\end{array}$ & 30 & 25 & 15 & 15 & tr. & 20 & 20 & & & \\
\hline
\end{tabular}




\section{Commentaires :}

Confrontée à la pédogénèse du lehm, l'évolution des sols de plateau présente les caractères suivants :

- Les sols sont moins épais que le lehm : ceci ne semble guère offrir de signification dans la mesure où la molasse est en général plus argileuse et moins poreuse que le loess (densités apparentes moyennes de la molasse et du loess : 1,5 et 1,3 ), donc beaucoup moins perméable.

- Les sols de plateau sont également de type brun calcique, plus nettement lessivés cependant que le lehm: les indices de distribution de l'argile atteignent 1,7 (plus bas pour le fer libre) et les revêtements argileux y sont plus nombreux. Comme le lehm, les sols sont toujours très calciques et souvent légèrement carbonatés, les complexes absorbants saturés, les $\mathrm{pH}$ basiques ou proches de la neutralité (sur molasse sableuse, profil 2): par leur ambiance ionique, ces " boulbènes de plateaux " contrastent singulièrement avec les véritables boulbènes des terrasses alluviales 2 .

- Sur molasse calcaire (profil 1), l'horizon d'accumulation des carbonates montre souvent, dans une pâte enrichie en calcaire diffus, des concrétions et poupées qui s'apparentent étonnamment à celles du loess, ainsi que des éléments de croûte feuilletée, très délabrée il est vrai. Nous n'avons jamais retrouvé de formes concrétionnées identiques dans la molasse profonde elle-même.

- En ce qui concerne les associations de minéraux argileux, le schéma évolutif est comparable à celui du lehm, mais en plus complexe, du fait de l'hétérogénéité de la molasse: diminution de la montmorillonite, apparition et renforcement des interstratifiés, maintien ou augmentation de l'illite, disparition de la chlorite uniquement en surface.

De ce qui précède, nous pouvons tirer deux enseignements importants :

$1^{\circ}$ Les sols de plateaux sont des sols encore jeunes, comme en témoignent leur profondeur modérée et la séquence évolutive des minéraux argileux. Ils sont somme toute, encore assez proches de leur matériel parental molassique. Pour observer la molasse stampienne vigoureusement altérée in situ, il faut se transporter non loin du Terrefort, sur la terrasse moyenne de la Garonne, vraisemblablement mindélienne. Là, sous 4 à $5 \mathrm{~m}$ d'alluvions, la molasse est transformée sur plusieurs mètres. Les pH sont très acides $(4,5$ à 5,5$)$, il n'y a plus trace de calcaire, le complexe est largement désaturé $(S / T$ de 10 à $60 \%$ ), la montmorillonite et la chlorite ont totalement disparu du stock argileux. A 2 ou 3 mètres sous l'alluvion, on retrouve un horizon d'accumulation calcaire, puis la molasse calcaire à montmorillonite, illite et chlorite. Le contraste est donc éclatant entre cette molasse altérée depuis le Mindel et les sols de plateau. Il n'est guère possible d'imaginer non plus que ces derniers se so:ent développés dans un éluvium ancien de la molasse: dans cette hypothèse, le cortège de minéraux argileux en fournirait immédiatement la preuve, ce qui n'est pas le cas.

2. Les sols de plateaux sont plus évolués que le lehm (profil textural, taux de libération du fer $\mathrm{Fe} \mathrm{L} / \mathrm{Fe} \mathrm{T} \%$ : en moyenne 50 à $70 \%$ contre 40 à $50 \%$ ). Ils semblent avoir subi de façon très nette le climat sec et froid qui a vu le

2. On observe cependant, au Nord-Est de Toulouse, des sols de plateau un peu plus lessivés et plus acides (pH 6 a 6,5). Ce dernier caractère est lié, semble-t-il, aux conditions légèrement hydromorphes (concrétions de fer) qui prévalent sur certains replats particulièrement étendus et aplanis. 
dépôt du loess : on ne peut s'expliquer autrement la présence des concrétions, poupées et éléments de croûte calcaire qui parsèment les horizons $\mathrm{C} \mathrm{Ca}$. De même, les fissures emplies de calcaire pulvérulent, que l'on observe surtout dans les premiers décimètres de la molasse, en sont-elles probablement aussi une manifestation (M. Mouline et al., 1969).

De tout cela, on retiendra donc essentiellement que les sols de plateau et, par voie de conséquence, les replats qui les portent, sont de formation récente quoique antérieurs à l'épisode éolien du Würm terminal. Dans ces conditions, il est clair que les éléments de plateau actuels n'ont plus rien de commun avec des lambeaux de surface pliocène ou quaternaire ancienne. De ceux-ci, ils ont certes probablement hérité le dessin général et surtout le caractère de niveaux dominants; ils. n'en ont toutefois conservé ni l'altitude, ni, a fortiori, le matériel de surface ${ }^{3}$.

Tout ceci nous porte à croire que le décalage altimétrique, précédemment évoqué, entre les hauts cailloutis garonnais et ariégeois et la surface culminante du Lauragais, n'appelle pas nécessairement une explication mobiliste par flexures et affaissements plio-quaternaires. On rejoint là, l'observation de F. TAILLEFER (1948, 1951), selon laquelle une véritable inversion du relief se serait produite dans la région au cours du Quaternaire, toutes les zones de cailloutis anciens s'étant peu à peu dégagées du paysage sous l'effet de l'érosion différentielle. Précisément, dans le Lauragais toulousain, tous les points hauts, buttes, croupes, replats, ont dû s'abaisser d'une ou plusieurs dizaines de mètres depuis le dépôt des hauts cailloutis alluviaux. Cela semble indiquer que le réseau hydrographique s'est installé très tôt au Quaternaire, découpant rapidement en lanières et replats le plateau originel, déblayant les matériaux arrachés aux éléments de plateau après chaque phase de ruissellement quelque peu importante.

Quant à la présence occasionnelle, sur les plateaux, de cailloutis quartzeux rougis et de lydiennes, elle s'interprète aisément si l'on sait que la molasse, formation détritique d'origine pyrénéenne, montre çà et là, dans sa masse, des lits ou des lentilles de même nature. Tous les autres faciès, en particulier l'argile rouge à éléments quartzeux, ne s'observent qu'à proximité immédiate des hautes nappes alluviales et en proviennent indiscutablement.

Peut-on préciser l'âge des sols de plateau et, par conséquent, celui du façonnement des replats? Les caractères de l'évolution pédologique, nous l'avons vu, plaident en faveur d'une période antérieure à l'épisode éolien du Würm supérieur. Probablement ce façonnement remonte-t-il à la première partie du Würm, comme semble en témoigner une phase de colluvionnement ancien colmatant les talwegs et que nous évoquerons un peu plus loin.

\section{IV. - LES DEPOTS COLluVIAUX ET LES SOLS (fig. 5)}

La mise en place des formations colluviales qui tapissent les versants longs est assurément récente : elles fossilisent en effet toutes les formes de creusement, excepté l'entaille actuelle des ruisseaux. Ces dépôts, qui offrent souvent l'aspect de coulées boueuses, ont été rapportés par $H$. ENJALBERT soit aux solifluxions par gel et dégel de la dernière période gaciaire, soit, pour celles d'entre elles qui co:ffent le loess, à la période immédiatement postérieure à l'épisode éolien.

3. Il semble qu'il n'en soit pas toujours de même dans le Terrefort tarnais : là, en effet, la proximité du Massif central et l'existence de hauts cailloutis tarnais sur certains replats, ont probablement autorisé la conservation de niveaux réellement anciens. 


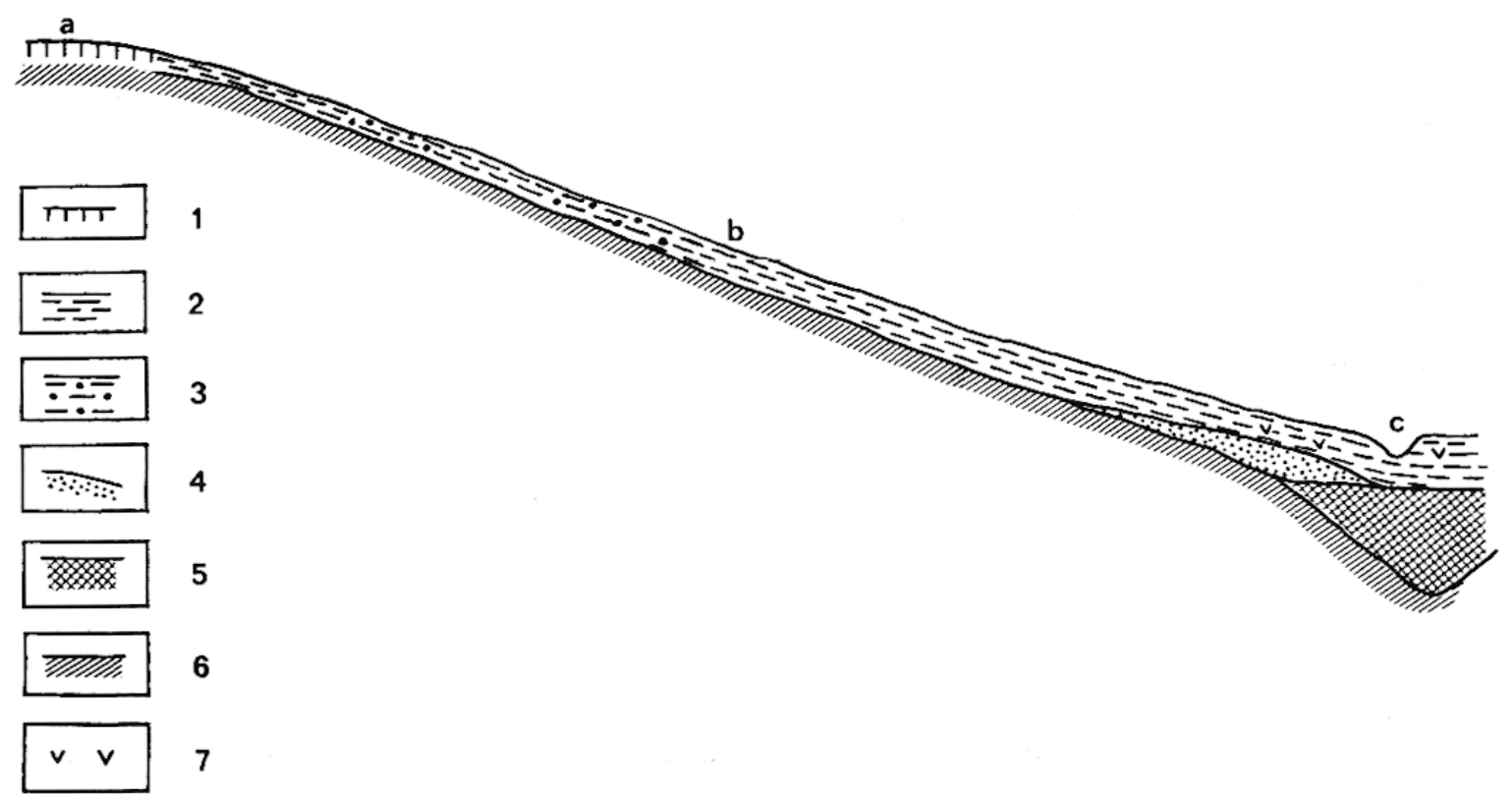

Fig. 5. - Séquence de sols sur versants longs.

a. Replat dominant. - b. Versant long. - c. Entaille récente des ruisseaux. - 1. Sols de plateau. - 2. Sols bruns calciques sur colluvium homogene argileux. - 3. Sols bruns calcaires sur colluvium grossier a éléments calcaires. - 4. Placage loessique fossilisé. - 5 . Colluvions anciennes très hétérogènes. - 6. Molasses. 7. Faciès hydromorphe.

Les sondages effectués dans les fonds de talwegs ont cependant montré une épaisseur souvent considérable $(5$ à $10 \mathrm{~m})$ de matériel colluvionné, enfoui sous les dépôts plus récents. Très hétérogène, composé pour partie de molasse remaniée de divers faciès, de fragments de grès calcaire et de dépôts argileux, ce matériel a livré un outillage fruste de type moustérien, rapporté au Würm ancien (A. CAvaille, 1969). Nous pouvons voir là une première génération de colluvions, antérieures à celles qui drapent les versants. Si l'on en juge par la puissance des dépôts qui ont subsisté au fond des talwegs ainsi que par leur composition (nombreux débris de molasse), cette phase de colluvionnement a dû être particulièrement active. Probablement est-ce à la même époque que se sont façonnés les replats actuels : ils se seront alors substitués à des aplanissements plus anciens et plus élevés, dont les sols et la molasse superficielle, largement décapés puis accumulés dans les fonds de vallon, constituent les dépôts obserrvés aujourd'hui.

Les matériaux récents qui tapissent les pentes sont en revanche beaucoup moins profonds (moins de $100 \mathrm{~cm}$ sur la partie amont des versants, de 100 à $200 \mathrm{~cm}$ à l'aval) mais de constitution plus homogène (" argile de coulière ", de G. ASTRE), généralement argileuse. On observe cependant, surtout à l'amont des versants, de fréquentes passées plus grossières : éléments de molasse peu altérée, fragment de grès tendre, concrétions calcaires analogues à celles des sols de plateau, tout ceci dispersé sans aucun ordre dans la masse du matériel. Ces formations reposent sur la molasse en place qu'ils ravinent par endroit. Notons également la présence régulière vers le bas des versants, d'une couche superficielle de 20 à $30 \mathrm{~cm}$ d'épaisseur, que tout désigne comme un apport récent lié à la mise en culture (fragments de brique et de poterie, pièces de métal, ossements d'animaux domestiques). Très localement, cette couverture récente atteint 1 ou $2 \mathrm{~m}$ d'épaisseur. 


\section{LES SOLS}

Dans l'ensemble, les sols sur colluvions récentes se rapprochent soit des sols bruns calcaires peu évolués (sur dépôts à éléments calcaires figurés des versants amont) soit, plus fréquemment, des sols bruns calciques (sur matériel argileux homogène). Au pied des versants, là où la pente est faible (5 à $10 \%)$, une certaine évolution du profil textural se manifeste, mais le sol reste toujours calcique et légèrement calcaire. Enfin, dans les talwegs, les faciès d'hydromorphie actuelle sont de règle (concrétions de fer, bigarrures...). Nous présentons ci-dessous très succinctement deux profils-types de sol brun-calcaire et de sol brun calcique peu lessivé.

\section{Profil 1. - Sol BRUn calcaire}

Situation : versant supérieur de pente $18 \%$ vers l'Est, altitude $215 \mathrm{~m}$. Chaumes de céréales.

$0-15 \mathrm{~cm} \quad$ : brun-jaune beige, texture moyenne argilo-sableuse, racines, strucAp ture grumeleuse, calcaire, quelques éléments calcaires grossiers ( * tête-de-chat ", concrétions dures), limites progressives avec (B);

$15-60$ : beige, texture moyenne sablo-argileuse très hétérogène, struc(B) ture motteuse, devenant compact en profondeur, très calcaire, puis nombreux éléments de calcaires grossiers dispersés dans la C masse (grès, concrétions), limites nettes avec la molasse, soulignées par une ligne de petits quartz et de concrétions en amas;

Plus de $60 \mathrm{~cm}$ : molasse stampienne argilo-calcaire à bariolage crangé et gris, molasse quelques veines de calcaires poudreux.

Profil 2. - Sol. brun calcioue lessivé

Situation: bas de versant, pente $6 \%$ vers le Nord-Est, altitude $190 \mathrm{~m}$. Affleurement loessique en profondeur. Chaumes de céréales.

0 - $30 \mathrm{~cm}$ : brun à brun-jaune 10 YR $4 / 4$ à 2,5 YR $5 / 4$, texture argileuse, Ap structure nuciforme à grumeleuse, racines nombreuses, fragments de briques, limites assez nettes avec (B);

$30-110 \mathrm{~cm}$ : brun à brun-jaune 10 YR $5 / 4$ à 2,5 YR 6/4, texture argileuse, (B) structure polyédrique nette, compact, limites nettes sur le puis C loess sous-jacent;

Plus de $110 \mathrm{~cm}$ : grège $2,5 \mathrm{Y} 7 / 4$, sableux, massif, vermicules et pseudomycéloess lium calcaires, très calcaire.

\section{Commentaires :}

Le caractère peu évolué de ces sols est l'indice de formations jeunes. Toutefois, le degré d'évolution ne constitue pas ici un critère chronologique très sûr: la pente favorise en effet à la fois le rajeunissement des sols (c'est là l'origine de la couverture anthropique des bas de versants) et le renouvellement des solutions calciques, ce qui contribue à ralentir aussi bien le lessivage que l'altération des minéraux phylliteux. A cet égard, la composition et la position de ces 
TABLEAU III

Données analytiques sommaires (profils 1 et 2)

\begin{tabular}{|c|c|c|c|c|c|c|c|c|c|}
\hline \multirow{2}{*}{$\begin{array}{l}\text { Profondeur } \\
\text { (cm) }\end{array}$} & \multirow{2}{*}{ A\% } & \multirow{2}{*}{$\underset{\mathrm{T}}{\text { Calcaire }}$} & \multirow{2}{*}{$\begin{array}{r}\text { pH } \\
\text { eau }\end{array}$} & \multirow{2}{*}{$S / T / Z$} & \multicolumn{5}{|c|}{ Minéraux argileux } \\
\hline & & & & & I & M & $\mathrm{K}$ & C & $14 C-14 V$ \\
\hline Profil 1 & & & & & & & & & \\
\hline $0-15$ & 20,7 & 5,3 & 8,5 & 100 & 20 & 40 & 15 & 5 & 20 \\
\hline $40-50$ & 18,9 & 10,1 & 8,4 & 100 & 25 & 40 & 15 & tr. & 20 \\
\hline $70-80$ & 22,9 & 11,7 & 8,7 & 100 & 25 & 50 & 20 & 5 & tr. \\
\hline Profil 2 & & & & & & & & & \\
\hline $10-20$ & 31,3 & 0,8 & 7,7 & 100 & 30 & 30 & 10 & & 30 \\
\hline $60-80$ & 36,8 & 0,7 & 8,1 & 100 & 20 & 30 & 20 & tr. & 30 \\
\hline $130-140$ & 6,3 & 28,3 & 8,0 & 100 & 25 & 55 & 15 & 5 & tr. \\
\hline
\end{tabular}

*Illite I, Montmorillonite M, Koolinite K, Chlorite C, interstrastifiés Chlorite - Vermiculite 14 C-14 V.

dépôts (recouvrement du loess) semblent plus probantes. Aussi bien, plus que l'âge de la formation, c'est son origine qu'il convient de souligner. Il est clair en effet qu'elle provient essentiellement du démantèlement partiel des replats dominants. Les profils des versants expriment parfaitement cette origine: les sols bruns calciques montrent une association de minéraux argileux qui reflète étonnamment la composition moyenne des sols de plateau (mélange des horizons). Les sols bruns calcaires, quant à eux, trahissent la complexité de leur matériau originel : fragments de molasse relativement fraîche, intimement mêlés aux débris des sols de plateau. Ils montrent par ailleurs des amas de concrétions calcaires qui ne peuvent provenir que des horizons d'accumulation calcaire des sols de replats. Cette observation constitue un argument de plus en faveur d'une mise en place très récente de ces dépôts, postérieure en tout cas à l'épisode du loess et du concrétionnement calcaire.

\section{V. - CONCLUSION : ESSAI DE CHRONOLOGIE}

En résumant les éléments présentés ci-dessus, on peut tenter de reconstituer à grands traits l'évolution du Lauragais toulousain au Quaternaire récent.

- Au début du Würm, plateaux et replats dominent un paysage de vallons et de versants. Ils ne représentent plus que le simple témoignage, très dégradé, de ce qui était autrefois un vaste plateau nettement plus élevé, entaillé de larges vallées à fond plat, disposition que suggère encore de nos jours l'étagement fré. quent des replats supérieurs et inférieurs.

- Au cours d'un épisode de ruissellement actif de la première moitié du Würm, les replats sont une fois encore démantelés et abaissés: décapage géné- 
ralisé de la surface et éboulements latéraux. Le matériel ainsi déblayé transite sur les pentes et est pris en charge par des ruisseaux assez vigoureux car bien alimentés, qui l'étalent et l'accumulent dans les talwegs ou, occasionnellement, l'évacuent plus loin vers les vallées plus importantes (on en trouve peu de traces, sauf sur le versant droit de l'Ariège et de la Garonne). Le retour à des conditions moins actives, probablement favorisées par une meilleure couverture végétale, facilite l'accumulation des dépôts dans les fonds de vallon où on les observe uncore de nos jours. C'est à cette époque que les sols des replats commencent à évoluer vers le type brun calcique lessivé: les horizons d'accumulation de calcaire diffus ou poudreux se constituent en profondeur.

- Dans la dernière partie du Würm s'installe un climat plutôt sec et froid. On imagine aisément un paysage où sur les versants dégarnis de végétation, la molasse est mise à nu et ravinée par de rares mais violents ruissellements concentrés dont la charge viendra s'étaler sur les dépôts épais antérieurement arcumulés dans les fonds de vallons. C'est sur ces versants que le vent va prélever les constituants du loess, dont le matériel argileux s'apparente étroitement à celui de la molasse fraîche, pour les déposer ensuite non loin de là, au creux des vallons et sur le versant gauche de la vallée de l'Hers Mort (vents dominants d'Ouest ? G. Astre, 1959). C'est à cette époque aussi que le calcaire du loess se réorganise en concrétions et tubulures: ce concrétionnement se manifeste également dans l'horizon calcaire des sols de plateau, sous l'action conjuguée des longues périodes de sécheresse et d'une végétation lâche mais à enracinement probablement profond (G. Beaudet et al., 1967; J. Hubschman, 1967; A. Rubllan, 1970). En ce sens, les sols qui tapissent actuellement les éléments de plateau sont, mais pour une part seulement (car ils continuent à évoluer), des paléosols, puisqu'ils portent la trace de conditions écologiques aujourd'hui révolues.

- Une nouvelle récurrence humide inaugure les temps holocènes: moins longue et moins marquée que la précédente, elle provoque le démantèlement partiel des éléments de plateau, dont seuls les secteurs les moins exposés et les mieux garnis de végétation semblent avoir subsisté dans le paysage actuel. Sols et éléments de molasse sont étalés pêle-mêle sur les versants que bien souvent ils ravinent, venant recouvrir à l'aval les dépôts plus anciens des fonds de vallon, et, localement, fossiliser le loess ${ }^{4}$. Sur les versants courts et raides, les colluvions ne font que transiter alors qu'elles s'accumulent peu à peu sur les pentes modérées. Tandis que les sols de plateau continuent leur évolution, ceux des versants longs se développent à leur tour très lentement vers le type brun calcaire ou brun calcique (non ou peu lessivé). Dans le même temps, l'hydromorphie qui affecte les fonds de vallons et parfois les pieds de versants, ne fait que prolonger celle que connurent probablement les périodes antérieures suffisamment humides.

- A partir de cette époque, l'organisation actuelle du paysage est pratiquement fixée, tandis que la végétation forestière va recouvrir croupes et versants. Seuls, la légère incision récente des ruisseaux, puis les défrichements médiévaux et la mise en culture actuelle, viendront y apporter quelques retouches : drapage des bas versants par un colluvium épais le plus souvent de 20 à $30 \mathrm{~cm}$; arrachements et foirages de versants, localisés au niveau de suintements ponctuels (A. Cavaillé, 1969).

En définitive, de l'ensemble des observations présentées dans ce travail, on peut dégager trois éléments principaux :

4. Le lœss enfoui ne montre jamais d'altération superficielle, soit qu'il ait été érodé au début du colluvionnement postérieur, soit que celui-ci ait été mis en place immédiatement après les dernières phases du dépôt éolien. 
- Le modelé du Lauragais toulousain est d'acquisition récente : en particulier, croupes et aplanissements dominants, généralement considérés comme les vestiges de surfaces très anciennes, ont été taillés à une époque récente.

- Les replats actuels constituent toutefois les formes les plus anciennes de cette région du Terrefort. Ces niveaux, de toute façon antérieurs à l'épisode éolien de la fin du Würm, ne sont cependant pas très vieux: ils datent probablement de la première moitié du Würm.

- Les colluvions qui drapent les versants du Terrefort sont, pour l'essentiel, postérieures à la phase de dépôt du loess. Elles tirent leur origine de la destruction partielle des éléments de plateau.

\section{BIBLIOGRAPHIE}

ASTRE (G.). - 1953 a. * L'argile grumeleuse de coulière, couverture quaternaire du haut terrefort toulousain $», 78^{\circ}$ Congrès soc. savantes, Sect. des Sc., p. 65-71.

Astre (G.). - 1953 b. « Mastodonte de Bourg-Saint-Bernard et érosions miocènes dans le bassin sous-pyrénéen $\star$, Bull. Soc. géol. de Fr., no 6, t. III, p. 253-260, Paris.

ASTRE (G.). - 1959. "Terrains stampiens du Lauragais et du Tolosan n, Bull. Soc. hist. nat. Toulouse, t. 94, p. 8-168.

Beauder (G.), Maurer (G.), RuellaN (A.). - 1967. " Le Quaternaire marocain. Obser. vations et hypothèses nouvelles ${ }^{\prime}$, Rev. de géogr. phys. et de géol. dyn., vol. IX, fasc. 4, p. 269-310, Paris.

Brunet (R.). - 1965. Les Campagnes toulousaines. Etude géographique, Toulouse, imp. F. Boisseau, 727 p. (thèse Lettres, Toulouse).

CAMEZ (T.). - 1962. Etude sur l'évolution des minéraux argileux dans les sols des régions tempérées. Mém. serv. Carte géol. Als.-Lor., t. 20, 90 p. (thèse Sciences, Strasbourg).

Cavaun (A.). - 1969. « Formations superficielles et sols des coteaux molassiques au sud-est de Toulouse », Livret-guide de l'excursion A6, VIII' Congr. INQA, p. 16-23, Paris.

Duchaufour (Ph.). - 1968. L'Evolution des sols, 94 p., Masson et $C^{\text {te }}$, Paris.

ENJalbert (H.). - 1960. Les Pays aquitains. Le modelé et les sols, t. I, Bordeaux. impr. Bière, 618 p. (thèse Lettres, Bordeaux.

Hubschman (J.). $\rightarrow$ 1967. Sols, pédogenèse et climats quaternaires dans la plaine des Triffa (Maroc), Toulouse, multigr., $152 \mathrm{p}$. (thèse doct.-ing. Sciences, Toulouse).

Hubschman (J.). - 1971. « Le lehm de Toulouse-Rangueil : signification pédologique et implications géochronologiques $\%$, Comm. $96^{\circ}$ Congrès Soc. savantes, Sect. Sc. (à paraître).

Mouline (M.), Birot (P.), Paquereau (M.). - 1969. "Le rebord Nord-Est de la Montagne Noire dans la région de Revel $"$, Livret-guide de l'excursion $A 6$, VIII' Congr. INQUA, p. 106-109, Paris.

RUBLLAN (A.). - 1970. Les sols a profil différencié des plaines de la Basse Moulouya (Maroc oriental), Strasbourg, multigr., 482 p. (thèse Sciences, Strasbourg).

TAlllEFER (F.). - 1948. " Le rôle morphologique des nappes d'alluvions caillouteuses du bassin aquitain $»$, C.R. Ac. des Sc., Paris, t. 227, p. 978-980.

TAllLefer (F.). - 1951. Le piémont des Pyrénées françaises. Contribution à l'étude des reliefs du piémont, Toulouse, Privat, $383 \mathrm{p}$. (thèse Lettres, Toulouse). 